Chapter 10

\title{
Muscle Fibers Lacking Desmin in the Extraocular Muscles: A Paradigm Shift
}

\author{
Fatima Pedrosa Domellöf \\ Additional information is available at the end of the chapter
}

http://dx.doi.org/10.5772/66928

\begin{abstract}
The extraocular muscles are highly specialized muscles responsible for the complex movements of the eyeball. They differ from other skeletal muscles in many respects, including fundamental components of the contractile apparatus and the extracellular matrix. Using immunohistochemistry and a battery of well-characterized antibodies, we have investigated the composition of the cytoskeleton of their myofibers with respect to desmin, vimentin, and nestin. In the adult and fetal human extraocular muscles, a subgroup of the slow tonic muscle fibers is lacking desmin. These fibers, which are multiply innervated, show a normal myofibrillar arrangement, maintained mitochondrial distribution, and sarcolemma integrity. Desmin, the most abundant intermediate filament protein in muscle, has been considered a ubiquitous protein in skeletal muscle fibers where it links adjacent myofibrils and the myofibrillar network to the sarcolemma, the mitochondria and the membrane of the nuclei. The functional implications of the lack of desmin remain to be determined, but these findings represent a paradigm shift, as desmin has been regarded a ubiquitous protein of the cytoskeleton of muscle fibers.
\end{abstract}

Keywords: desmin, human, extraocular muscle, myosin heavy chain slow tonic, multiple innervation

\section{Introduction}

The extraocular muscles differ significantly from other muscles in the body, including their structural composition, physiological properties, and response to disease [1-3]. Because the extraocular muscles are more resistant to a number of diseases than the other muscles [3], it has been hypothesized that a better understanding of their composition may provide useful clues to develop strategies to face the challenge posed by muscle dystrophies and other neuromuscular 
diseases. We have studied the composition of the human extraocular muscles with respect to the myosin heavy chains (MyCHs) [4], the major determinants of contraction velocity and force; SERCA-1 and SERCA-2 (sarco/endoplasmic endoreticulum calcium ATPase) [5], two important calcium transportation proteins; laminins [6], major components of the basement membrane and a number of proteins relevant for the neuromuscular junctions, e.g., gangliosides [7]. Because data on the composition of the cytoskeleton in the human extraocular muscles were lacking and because defects in the desmin gene cause myopathy and cardiomyopathy, we recently also investigated the distribution of the major intermediate filament proteins, desmin, vimentin, and nestin in the human adult extraocular muscles, using immunohistochemistry in serial sections and in 1- $\mu \mathrm{m}$ thick sections [8].

Desmin has been regarded as a ubiquitous protein in skeletal muscle fibers, being the first muscle-specific protein detected during development [9]. Desmin is the most abundant intermediate filament protein in muscle fibers, forming a three-dimensional scaffold along the entire muscle fiber. Desmin links adjacent myofibrils at the Z-discs, and it links the most peripheral myofibrils to the sarcolemma and to the membrane of the nuclei. It also links the myofibrils to other organelles, such as the mitochondria [10-12].

In spite of being the first muscle-specific protein expressed early during muscle development and being so abundant in mature muscle fibers, desmin is not strictly necessary for myogenesis or muscle fiber maturation (reviewed in Refs. [13, 14]). Desmin knockout (KO) mice develop rather normally, but they develop a cardiomyopathy and/or a skeletal myopathy that predominantly affects highly used muscles, such as the diaphragm. Early muscle differentiation appears normal but with time signs of pathology develop. The affected myofibers show misaligned myofibrils with the loss of anchorage to the sarcolemma and mitochondrial abnormalities. Data from the desmin $\mathrm{KO}$ animals indicate that desmin is essential for the integrity of highly used skeletal muscle and for proper mitochondrial morphology and positioning but that it is not essential for muscle development. In contrast, these desmin $\mathrm{KO}$ animals show affected regeneration capacity, with adipocyte accumulation and abnormal neuromuscular junction morphology. Furthermore, in this animal model, desmin seems to be necessary for proper excitation-contraction coupling and respiratory function [13, 14].

Patients with defects in the desmin gene develop progressive skeletal myopathy and cardiomyopathy, although the age of onset of the disease may be rather high. Desmin-related myopathies, which may be familial or sporadic, are generally characterized by the formation of abnormal desmin aggregates and have heterogeneous clinical hallmarks, which may include neuropathy and smooth muscle involvement, in addition to skeletal muscle and heart symptoms [13].

The complexity of actions performed by the extraocular muscles is reflected in their distinct fiber type composition and structural organization that differ substantially from ordinary skeletal muscles [1,2]. The extraocular muscles are among the fastest muscles in the human body, yet they are extremely fatigue-resistant. Their muscle fibers are organized into two layers: the orbital layer, closest to the orbital wall, and the global layer, facing the bulb. The muscle fibers in the orbital layer have a smaller diameter and do not extend the full length of the muscle. The muscle fibers in the extraocular muscles have small diameters, are loosely arranged in a bed of connective tissue, are richly supplied by capillaries, and belong to very small muscle units. 
The fiber type composition of the human extraocular muscles is very complex [4, 15]. Typically, the muscle fibers in the human extraocular muscles contain several myosin heavy chain (MyHC) isoforms, including isoforms not typically found in mature skeletal muscle such as MyHC embryonic and fetal, MyHC alpha cardiac, MyHC extraocular, and MyHC slow tonic and the composition of the muscle fibers varies along their length [4, 15]. A particular fiber type contains MyHC slow tonic, and it is multiply innervated. In the orbital layer, these fibers also have a twitch motor endplate in the middle portion, whereas those in the global layer seem to be tonic along their entire length (reviewed in Refs. [1, 2]).

The gene expression profile of the extraocular muscles differs fundamentally from that of the limb muscles with respect to metabolic pathways, structural components, developmental and regeneration markers, by over 300 genes [16]. Indeed, the extraocular muscles are classified as a separate muscle allotype (a class of muscles), in contrast to the other allotypes: (i) the limb and trunk muscles and (ii) the masticatory muscles [17].

The extraocular muscles also differ from the other muscles in the human body by their particular response to disease [3]. The extraocular muscles are selectively affected in autoimmune disorders such as myasthenia gravis, Miller-Fisher syndrome, and Grave's ophthalmopathy. In contrast, they are strikingly spared in various muscular dystrophies such as Duchene and Becker muscular dystrophy, limb-girdle, and congenital muscular dystrophies that are devastating for the other muscles in the body, by causing severe weakness, loss of ambulation, and early death [18].

Here, the present state of knowledge regarding the composition of the cytoskeleton of human extraocular muscles with respect to desmin, vimentin, and nestin is reviewed [8].

\section{Lack of desmin in fibers of the human extraocular muscles}

The vast majority of muscle fibers in the extraocular muscles contain desmin (Figure 1), as expected, but the muscle fibers containing MyHC slow tonic show different staining patterns concerning desmin, irrespective of whether they are present in the orbital or global layers [8]. Some of these muscle fibers apparently lack desmin completely (Figure 1), both at the subsarcolemmal level and in between myofibrils, as they are completely unstained by a battery of mono and polyclonal antibodies against desmin.

In thin sections $(1 \mu \mathrm{m}$, Figure 2$)$, a normal myofibrillar organization of these muscle fibers is apparent and antibodies against MyHC slow tonic and/or MyHC slow label them, but they are completely unlabeled by different antibodies against desmin. Three additional staining patterns are present among the muscle fibers containing MyHC slow tonic in the human adult extraocular muscles: (i) muscle fibers that show desmin only subsarcolemmally; (ii) muscle fibers with desmin staining present both between myofibrils and below the sarcolemma, but with extremely weak staining intensity and (iii) muscle fibers showing a typical desmin cytoskeleton pattern, as shown in the other muscle fibers (Figure 2). Moreover, the lack of desmin in the myofibers containing MyHC slow tonic is already present in fetal human extraocular muscles at 16 and 18 weeks of gestation. The pattern of distribution of mitochondria is maintained in all adult muscle fibers [8]. 

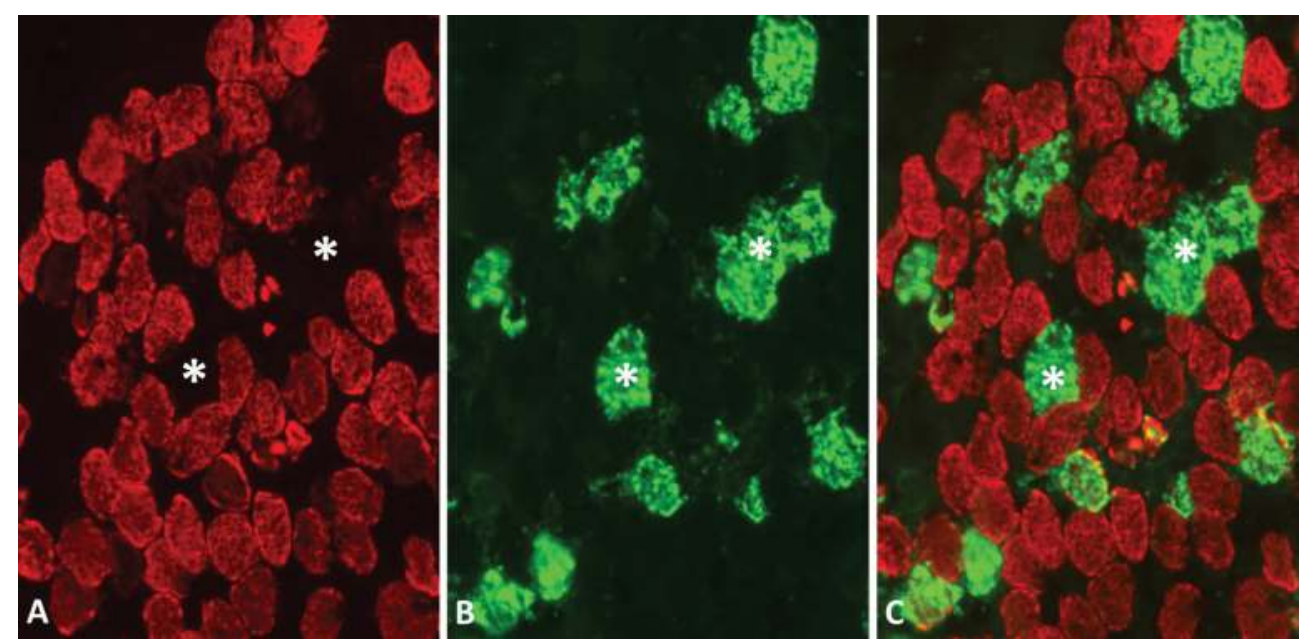

Figure 1. Cross-section (5 $\mu \mathrm{m}$ ) of adult human extraocular muscle double-stained for desmin (A, red) and MyHC slow (B, green). The merged picture of A and B is shown in C. Examples of muscle fibers lacking desmin are marked with an asterisk.
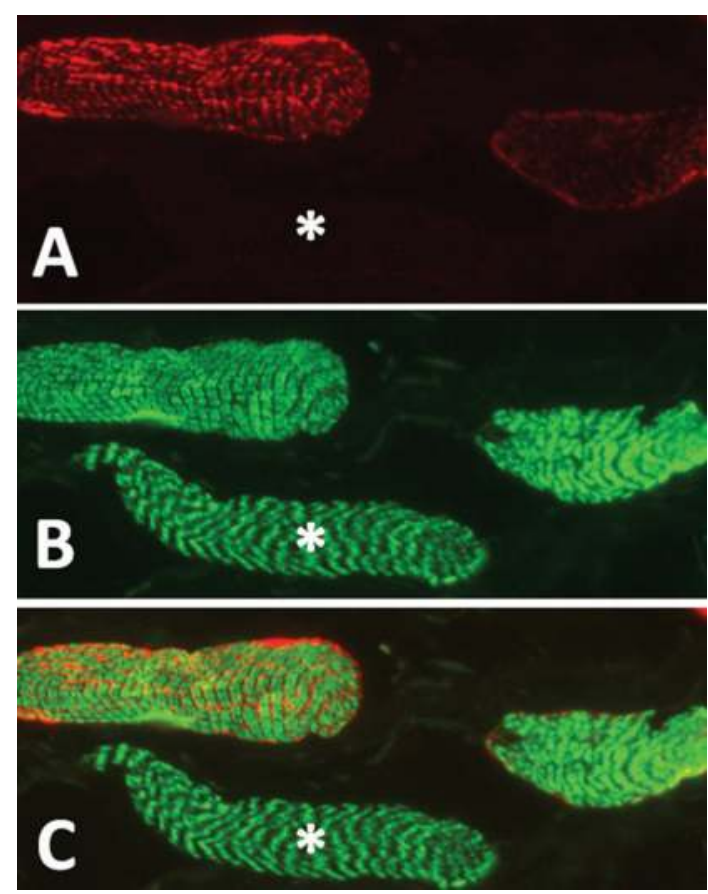

Figure 2. Thin section $(1 \mu \mathrm{m})$ of adult human extraocular muscle double-stained for desmin (A, red) and MyHC slow tonic (B, green), showing a muscle fiber lacking desmin (asterisk) both in between myofibrils and under the sarcolemma. A merged picture with both stainings is shown in C. The typical distribution of desmin is seen in the other two muscle fibers shown. Notice that all three muscle fibers contain MyHC slow tonic. 
Nestin does not appear to substitute for desmin in the desmin-negative muscle fibers, although nestin is present in a very large number of normal adult muscle fibers in the human extraocular muscles. Vimentin is not present in any muscle fibers in the adult human extraocular muscles [8].

More recently, the absence of desmin has also been reported in slow muscle fibers of the uvula and palatopharyngeal muscles [19]. These fibers contain MyHC slow but lack MyHC slow tonic and they are also unstained with antibodies against the C-terminal of dystrophin. Similarly to the extraocular muscles, nestin and vimentin do not compensate for the lack of desmin in these muscle fibers [19]. The uvula and palatopharyngeal muscles are highly specialized and unusual in that they lack a firm bony attachment at least at one end and the authors suggest that the lack of desmin in a subgroup of the fibers in these two muscles likely reflects specialization to meet the complex requirements of oropharyngeal functions [19].

The presence of normal adult muscle fibers lacking desmin is a paradigm shift as this cytoskeletal protein has been regarded as ubiquitous in normal skeletal muscle fibers. Because the absence or presence of only trace amounts of desmin in muscle fibers of the human extraocular muscles is confirmed with a polyclonal antibody and two different monoclonal antibodies, in both 5 and $1 \mu \mathrm{m}$ sections, it is not likely that the findings are due to differences in epitope availability.

The absence of desmin in $\mathrm{KO}$ animals primarily leads to pathological changes in highly used muscles $[13,14,20]$. The extraocular muscles are also highly used muscles, but it may be so that the muscle fibers containing $\mathrm{MyHC}$ slow tonic and lacking desmin are used in a different pattern. These muscle fibers are typically multiply innervated, have a sustained mode of contraction, and they have been suggested to play a role in proprioception [9]. The fact that (i) no abnormalities in the pattern of mitochondria distribution are present in the fibers lacking desmin in the human extraocular muscles, along with (ii) a maintained myofibrillar organization, (iii) maintained fiber integrity, and (iv) the early establishment of this pattern during development [8] strongly suggest that the lack of desmin is indeed a special feature of these muscle fibers. These findings really represent a paradigm shift, as desmin can no longer be considered ubiquitous in muscle fibers and its ascribed fundamental role anchoring the mitochondria apparently does not apply to all muscle fibers.

Desmin is a crucial cytoskeletal protein in the vast majority of muscle fibers, and it is proposed to be fundamental for maintenance of fiber integrity, force transduction, and mechanochemical signaling as it links the contractile elements both to the sarcolemma and to the nuclear membrane $[14,21]$. However, it is difficult to speculate on possible physiological consequences of the absence of desmin on these slow tonic muscle fibers of the human extraocular muscles, and further studies correlating structural properties and physiological behavior are required.

\section{Conclusions}

Against all previous knowledge, desmin is lacking in a subgroup of normal myofibers in the human eye muscles [8]. Furthermore, nestin does not compensate for the lack of desmin, but an important number of muscle fibers normally contains nestin in these adult muscles. 
The finding of normal muscle fibers lacking desmin in adult and fetal human extraocular muscle represents a paradigm shift as desmin has been regarded as ubiquitous in muscle fibers. The presence of nestin in normal mature muscle fibers further emphasizes that the extraocular muscles differ fundamentally from the other muscles in the body.

\section{Acknowledgements}

Supported by grants from the Swedish Research Council, the County Council of Västerbotten (Cutting Edge Medical Research and Central ALF) and Stiftelsen Kronprinsessan Margaretas Arbetsnämnd för Synskadade.

\section{Author details}

\section{Fatima Pedrosa Domellöf}

Address all correspondence to: fatima.pedrosa-domellof@umu.se

Department of Clinical Sciences, Ophthalmology, Umeå University, Umeå, Sweden

\section{References}

[1] Sadeh M. Extraocular muscles. In: Engel AG, Franzini-Armstrong C, editors. Myology. New York: McGraw-Hill; 2004. pp. 119-127. DOI: 10.1002/ana.410360536

[2] McLoon LK, Christiansen SP. Extraocular muscles: Extraocular muscle anatomy. In: Dartt DA, Besharse JC, Dana R, editors. Encyclopedia of the Eye, Vol. 2. Oxford: Academic Press; 2010. pp. 89-98. DOI: 10.1016/b978-0-12-374203-2.00274-8

[3] Pedrosa Domellöf F. Extraocular muscles: Extraocular muscle involvement in disease. In: Dartt DA, Besharse JC, Dana R, editors. Encyclopedia of the Eye, Vol. 2. Oxford: Academic Press; 2010. pp. 99-104. DOI: 10.1016/B978-0-12-374203-2.00279-7

[4] Kjellgren D, Thornell LE, Pedrosa-Domellöf F. Myosin heavy chain isoforms in human extraocular muscles. Invest Ophthalmol Vis Sci. 2003;44:1419-1425. DOI: 10.1167/ iovs.02-0638

[5] Kjellgren D, Stal P, Larsson L, Furst D, Pedrosa-Domellof F. Uncoordinated expression of myosin heavy chains and myosin-binding protein $C$ isoforms in human extraocular muscles. Invest Ophthalmol Vis Sci. 2006;47:4188-4193. DOI:10.1167/iovs.05-1496

[6] Kjellgren D, Thornell LE, Virtanen I, Pedrosa-Domellof F. Laminin isoforms in human extraocular muscles. Invest Ophthalmol Vis Sci. 2004;45:4233-4239. DOI:10.1167/iovs. 04-0456

[7] Liu JX, Willison HJ, Pedrosa-Domellof F. Immunolocalization of GQ1b and related gangliosides in human extraocular neuromuscular junctions and muscle spindles. Invest Ophthalmol Vis Sci. 2009;50:3226-3232. DOI: 10.1167/iovs.08-3333 
[8] Janbaz AH, Lindström M, Liu JX, Pedrosa Domellöf F. Intermediate filaments in the human extraocular muscles. Invest Ophthalmol Vis Sci. 2014;55:5151-5159. DOI: 10.1167/ iovs.14-14316

[9] Furst DO, Osborn M, Weber K. Myogenesis in the mouse embryo: differential onset of expression of myogenic proteins and the involvement of titin in myofibril assembly. J Cell Biol. 1989;109:517-527. DOI: 10.1083/jcb.109.2.517

[10] Small JV, Furst DO, Thornell LE. The cytoskeletal lattice of muscle cells. Eur J Biochem. 1992;208:559-572. DOI: 10.1111/j.1432-1033.1992.tb17220.x

[11] Tokuyasu KT, Dutton AH, Singer SJ. Immunoelectron microscopic studies of desmin (skeletin) localization and intermediate filament organization in chicken skeletal muscle. J Cell Biol. 1983;96:1727-1735. DOI: 10.1083/jcb.96.6.1727

[12] Fuchs E, Weber K. Intermediate filaments: structure, dynamics, function, and disease. Ann Rev Biochem 1994;63:345-382. DOI: 10.1146/annurev.bi.63.070194.002021

[13] Paulin D, Li Z. Desmin: a major intermediate filament protein essential for the structural integrity and function of muscle. Exp Cell Res. 2004;301:1-7. DOI: 10.1016/j. yexcr.2004.08.004

[14] Capetanaki Y, Bloch RJ, Kouloumenta A, Mavroidis M. Psarras S. Muscle intermediate filaments and their links to membranes and membranous organelles. Exp Cell Res. 2007;313:2063-2076. DOI: 10.1016/j.yexcr.2007.03.033

[15] Wasicky R, Ziya-Ghazvini F, Blumer R, Lukas JR, Mayr R. Muscle fiber types of human extraocular muscles: a histochemical and immunohistochemical study. Invest Ophthalmol Vis Sci. 2000;41:980-990. Apparently there is no DOI for this article. This is the link to the article: http://iovs.arvojournals.org/article.aspx?articleid=2123010

[16] Fischer D, Bakay M, Gorospe JR, Kjellgren D, Pedrosa-Domellöf F, Hoffman E, Khurana TS. Definition of the unique human extraocular muscle allotype by expression profiling. Physiol Genomics. 2005;22:283-291. DOI: 10.1152/physiolgenomics.00158.2004

[17] Hoh JF, Hughes S, Hoy JF. Myogenic and neurogenic regulation of myosin gene expression in cat jaw-closing muscles regenerating in fast and slow limb muscle beds. J Mus Res Cell Motil. 1988;9:59-72. DOI: 10.1007/bf01682148

[18] Emery AEH. The Muscular Dystrophies. Oxford: Oxford University Press; 2001. 330 p. DOI: 10.1016/S0960-8966(03)00006-3 DOI:10.1016/S0960-8966\%2803\%2900006-3

[19] Shah F, Berggren D, Holmlund T, Levring Jäghagen E, Stål P. Unique expression of cytoskeletal proteins in human soft palate muscles. J Anat. 2016;228:487-494. DOI: 10.1111/ joa.12417

[20] Carlsson L, Thornell LE. Desmin-related myopathies in mice and man. Acta Physiol Scand. 2001;171:341-348. DOI: 10.1046/j.1365-201x.2001.00837.x

[21] Goldfarb LG, Olive M, Vicart P, Goebel HH. Intermediate filament diseases: desminopathy. Adv Exp Med Biol. 2008;642:131-164. DOI: 10.1007/978-0-387-84847-1_11 
